\title{
What Happens to Neonates Discharged After Management of Birth Asphyxia?
}

\section{Somashekhar Nimbalkar ${ }^{1}$}

Dimple Patel ${ }^{1}$, Vallaree Morgaonkar ${ }^{1}$, Nitesh Sadhwani ${ }^{1}$, Mayur Shinde ${ }^{2}$, Department of Pediatrics, Pramukhswami Medical College, Karamsad, Gujarat, India. Pin -388325

Central Research Services, Charutar Arogya Mandal, Karamsad, Gujarat, India. Pin - 388325

Email: somu somu@yahoo.com. Phone: 9825087842

\section{INTRODUCTION}

Birth asphyxia is defined as a delay in establishing spontaneous breathing/crying immediately after birth of the newborn.

Hypoxic Ischemic Encephalopathy (HIE) is the term commonly used to describe the neurological syndrome that occur following perinatal asphyxia. It is usually caused by severe birth asphyxia with secondary cerebral ischemia. It is an important cause of permanent damage to the Central Nervous System (CNS) which may result in neonatal death or manifest later as cerebral palsy or mental deficits. There is dearth of studies in India especially regarding long term follow up of infants of HIE due to birth asphyxia. Also, due to neuronal plasticity of brain in first two years of life, early follow up and treatment can diagnose neurological/visual/hearing deficits and give scope to provide supportive care and improve outcome.

So need for this study to know outcome of birth asphyxia so also improve quality of care and to give them a better quality of life.

\section{OBJECTIVE}

We administered questionnaire to parents of neonates who were discharged with a diagnosis of BA and ascertained their current medical status.

\section{Materials and Method:}

Patient admitted in Shree Krishna Hospital NICU in neonatal period due to BA, and discharged alive from NICU were assessed at follow up. Parents of 50 children from 2014 to 2016 were approached. Descriptive statistics (mean SD, frequency percentage) was used.$$
45
$$$$
20
$$

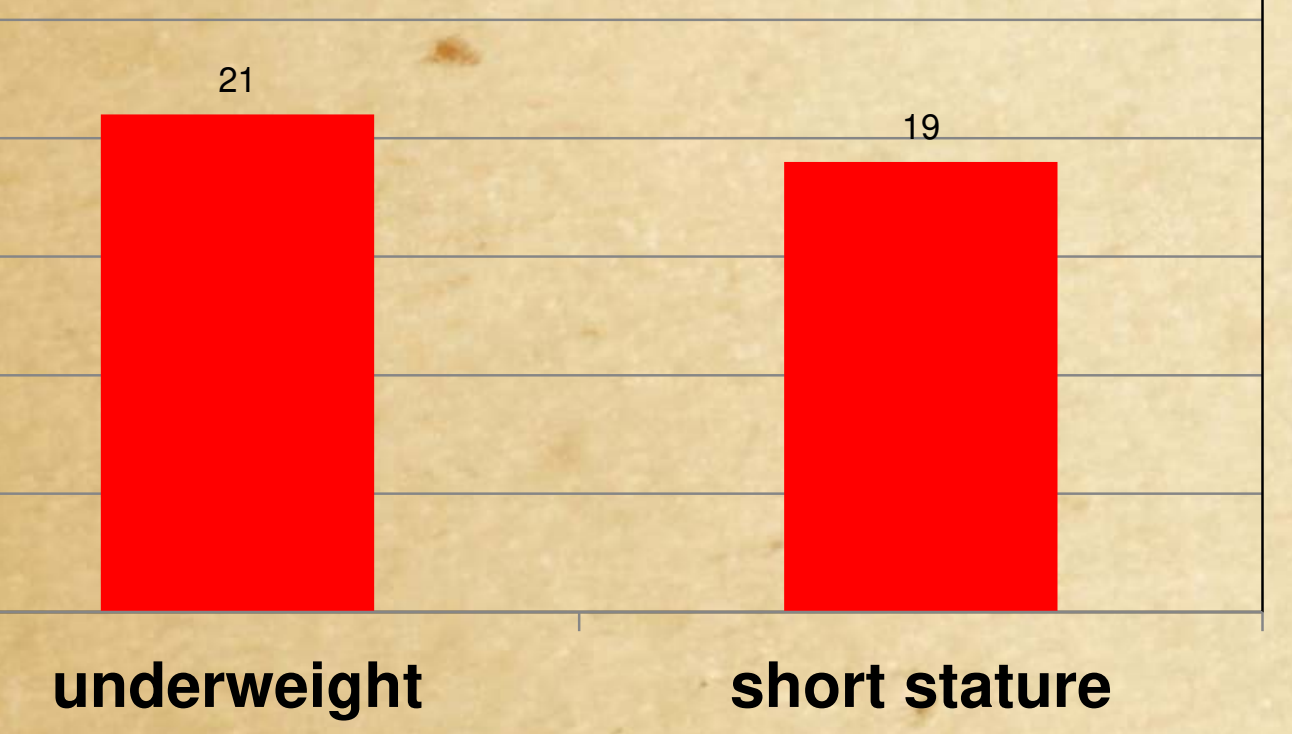

45

40

35

30

25

20

15

10

5

0

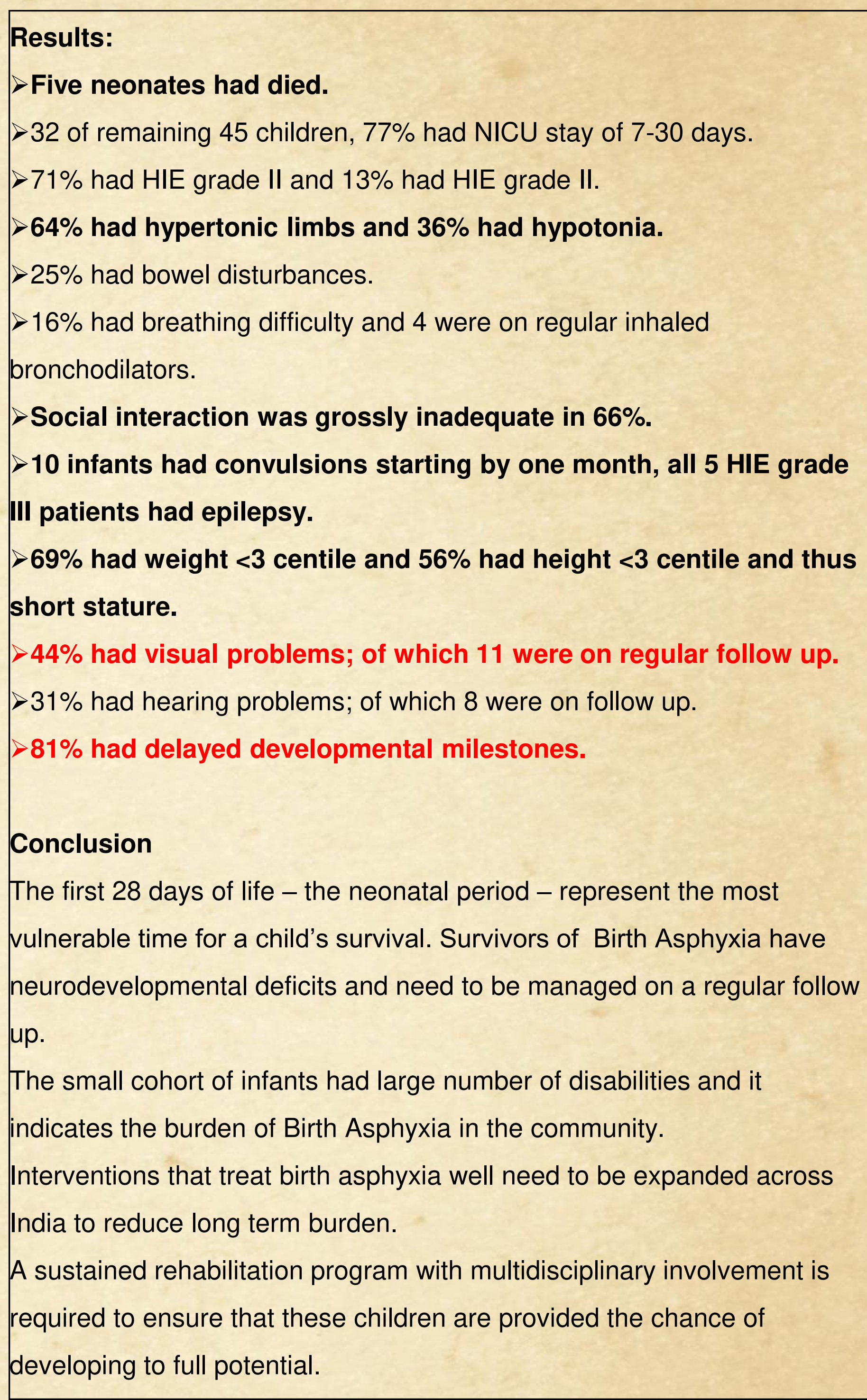

14

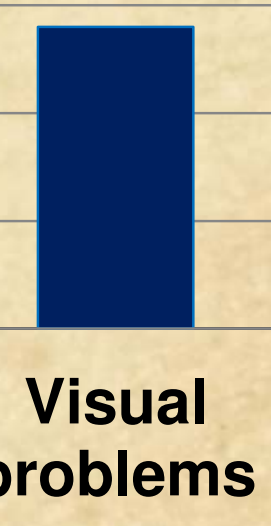

10

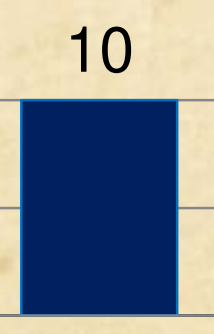

Convulsion problems

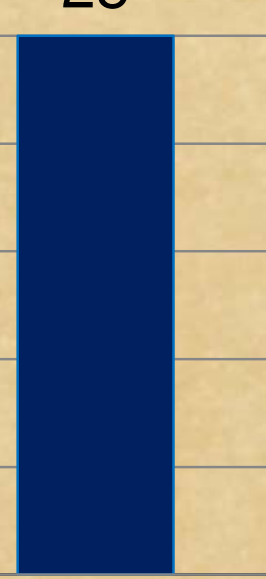

Delayed Hearing problems 